\title{
ÚLTIMOS AVANCES CIENTíFICOS DE LOS EFECTOS NEUROPSICOLÓGICOS DE LA EDUCACIÓN MUSICAL
}

LATEST SCIENTIFIC ADVANCES IN THE NEUROPSYCHOLOGICAL EFFECTS OF MUSIC EDUCATION

Katya Martin-Requejo

Sandra Santiago-Ramajo 
RECIBIDO: $26-05-2021$

ACEPTADO: $25-10-2021$

\section{RESUMEN}

La neurociencia y neuropsicología han ido evidenciando los beneficios de la música a nivel neuroanatómico y neurofuncional, así como sobre el rendimiento académico de los alumnos. La mayoría de estas evidencias se han obtenido mediante estudios retrospectivos basados en el estudio de personas con formación musical. Por ello, y contemplando que el impacto de la música es mayor durante la infancia, cada vez son más los estudios experimentales que, implementando programas musicales, están demostrando los beneficios causales sobre procesos neuropsicológicos y sobre el rendimiento académico. Sin embargo, los resultados continúan siendo poco concluyentes y faltan evidencias respecto al efecto de una educación musical no instrumental y creativa en niños de la etapa de Primaria. Conocer las relaciones de causalidad implicadas resultaría muy valioso para promover, desde el ámbito educativo, el diseño e implementación de programas musicales transversales fundamentados en conocimientos científicos, con el objetivo de incidir sobre los procesos neuropsicológicos subyacentes en el aprendizaje y mejorar así, el rendimiento académico de niños en etapa escolar.

\section{PALABRAS CLAVE}

\section{Músicajy, Neurøpsicología, Neurodesarrollo, Educación}

\section{A B S T R ACT}

Neuroscience and neuropsychology have been showing the benefits of music at a neuroanatomical and neurofunctional level, as well as on the academic performance of students. Most of this evidence has been obtained through retrospective studies based on the study of people with musical training. Therefore, and considering that the impact of music is greater during childhood, there are more and more experimental studies that, implementing musical programs, are demonstrating the causal benefits on neuropsychological processes and on academic performance. However, the results continue to be mixed and there is a lack of evidence regarding the effect of a non-instrumental and creative music education on children in the primary stage. Knowing the causal relationships involved would be very valuable to promote, from the educational field, the design and implementation of transversal music programmes based on scientific knowledge, with the aim of influencing the neuropsychological processes underlying learning and thus, improving the academic performance of school-age children.

\section{KEYWORDS}

Music, Neuropsychology, Neurodevelopment, Education 


\section{INTRODUCCIÓN}

En las últimas décadas ha aumentado el interés por el estudio del impacto de la música, evidenciando así modulaciones a nivel estructural y funcional en el cerebro de los músicos (Tanaka y Kirino, 2018). Junto con ello, diversos autores han demostrado que la formación musical también genera transferencias cognitivas lejanas, las cuales, a su vez, promueven mejoras en el rendimiento académico de los músicos (Swaminathan, 2018). La mayoría de estos estudios cuentan con un diseño ex post facto comparando los músicos con los no músicos, lo cual no permite conocer las relaciones de causalidad subyacentes en los beneficios atribuidos a la educación musical (Moreno y Bidelman, 2014). Ante dicha situación, diversos autores han iniciado el estudio de las causas implicadas a través de diseños experimentales $y$, teniendo en cuenta que el inicio temprano en la formación musical ha demostrado mayores beneficios anatomofuncionales en los músicos, cada vez es mayor el interés por comprender el impacto de intervenciones musicales en la infancia. Las evidencias hasta ahora obtenidas presentan gran heterogeneidad, al diferir, principalmente, en distintos aspectos metodológicos, lo cual pone de manifiesto la necesidad de continuar investigando en este ámbito con el objetivo de elucidar las inconsistencias observadas. Partiendo de este contexto, el presente artículo de revisión tiene como objetivo recoger las principales evidencias de los últimos 10 años respecto al impacto de la educación musical en procesos neuropsicológicos y en habilidades académicas de los niños, y destacar las necesidades para futuras líneas de investigación. Para ello, se parte de una revisión de las evidencias observadas en los músicos justificando la importancia del inicio temprano en la formación musical, para continuar con las evidencias existentes en relación a la infancia y terminar con las implicaciones que todo ello puede tener sobre el ámbito educativo.

\section{NEUROCIENCIA Y NEUROPSICOLOGÍA MUSICAL}

Los avances en las técnicas de neuroimagen han aumentado el interés por estudiar el impacto de la música sobre el cerebro y el desarrollo del ser humano desde el ámbito de la neurociencia cognitiva o la neuropsicología (Galera Núñez y Tejada Giménez, 2012; Kraus et al., 2014; Pearce y Rohrmeier, 2012). Ambas disciplinas científicas se enmarcan dentro de la neurociencia conductual, ya que estudian el sistema nervioso y su implicación en la capacidad cognitiva y conductual del ser humano (Boone y Piccinini, 2016; Portellano, 2010). Para ello, la neurociencia cognitiva utiliza las técnicas de neuroimagen funcional, mientras que la neuropsicología emplea test y pruebas neuropsicológicas para inferir el funcionamiento cognitivo (Portellano, 2008). Teniendo en cuenta que todo proceso mental y actividad sensomotriz implica una actividad neuronal subyacente (Maureira, 2010), y a fin de determinar unos modelos explicativos que favorezcan la comprensión de las interrelaciones entre la genética, las experiencias específicas y el desarrollo humano (Penhune, 2011), resulta esencial continuar investigando la relación entre las bases biológicas y la conducta humana.

Así pues, y atendiendo a su universalidad, su origen innato y su poder para involucrar amplios procesos cerebrales y estructuras cognitivas de forma simultánea, la música es un valioso ámbito de estudio para la neurociencia y la neuropsicología (Galera Núñez y Tejada Giménez, 2012; Kraus, Hornickel, et al., 2014; Pearce y Rohrmeier, 2012). Desde la perspectiva musical, estas dos ciencias estudian los aspectos biopsicológicos y los procesos cognitivos y emocionales implicados en la percepción y ejecución musical (Koelsch, 2009) y en este sentido, se ha evidenciado que la actividad musical, al implicar una alta precisión sensorial y cognitiva, requiere de la intervención de amplias estructuras cerebrales y habilidades superiores que, a su vez, inciden sobre los distintos procesos de aprendizaje (Herholz y Zatorre, 2012; Moreno y Bidelman, 2014; Sanju y Kumar, 2016). Asimismo, tanto la participación musical pasiva (recepción) como la activa (ejecución) parecen impactar en el cerebro tanto a nivel cognitivo como emocional (Benítez et al., 2018).

\section{IMPACTO DEL ENTRENAMIENTO MUSICAL FORMAL PROLONGADO}

Diversos estudios han aportado evidencias sobre los cambios estructurales y funcionales que el entrenamiento musical prolongando genera en el cerebro de los músicos (Jentschke et al., 2014; Marco-Pallarés et al., 2016). A nivel estructural, se han hallado diferencias neuroanatómicas en regiones fronto-temporales superiores y dorsolaterales, hipocampo, cuerpo 
calloso, corteza cingulada anterior bilateral, región parahipocámpica derecha y en el giro temporal medial posterior derecho (Burunat et al., 2015; James et al., 2017). La práctica musical prolongada, por lo tanto, induce un aumento en la neuroplasticidad estructural, a la vez que también aumenta la densidad de materia gris en amplias regiones cerebrales (Fauvel et al., 2014; James et al., 2013). En cuanto a la neuroplasticidad funcional, los músicos presentan una mayor simetría funcional en las redes visuales, motoras y somatosensoriales de los lóbulos parietales y frontales (Burunat et al., 2015), mejoras en los procesos de codificación neuronal auditiva (Bidelman y Krishnan, 2014), mayor integración entre redes motrices y perceptivas (Burunat et al., 2017), un aumento de actividad neuronal en el giro temporal superior derecho (Saari et al., 2018) y mejoras en la inhibición interhemisférica (VoIlmann et al., 2014).

Junto con ello, diversos estudios señalan que todas estas ventajas anatomofuncionales también inciden en otros procesos gracias a las transferencias cognitivas, promoviendo mejoras en la coordinación e integración visomotriz, reconocimiento de patrones visuales, funciones ejecutivas, memoria de trabajo, semántica, autobiográfica y episódica, y mejoras en habilidades lingüísticas y matemáticas y en el rendimiento escolar (Burunat et al., 2017; Fauvel et al., 2014; James et al., 2013; Linnavalli et al., 2018; Slater et al., 2017; Winner et al., 2013). Cabe destacar que, según señalan varios estudios, estas modulaciones cerebrales se ven influenciadas por la edad en la que se inicia el entrenamiento musical, considerando la primera infancia como el periodo más sensible (Bailey et al., 2012; Habibi et al., 2016; Schlaug et al., 2014; Skoe y Kraus, 2013).

\subsection{Beneficios de iniciar la formación musical a temprana edad}

En la primera infancia, el cerebro se encuentra en vías de desarrollo, por lo que iniciar una formación musical en dicho periodo hace que se maximicen los cambios cerebrales a través de la neuroplasticidad modulada por la experiencia (Skoe y Kraus, 2013). Esto es debido a que, durante los primeros años de vida, el cerebro tiene una mayor plasticidad, lo cual favorece una mejor integración de la información motriz y sensorial (Filippetti, 2011 ; Penhune, 2011 ). De este modo, la actividad musical temprana tiene un mayor impacto biológico en el cerebro de los niños, ya que incide sobre la neuroplasticidad rápida, modulando la codificación y funcionalidad auditiva, y favoreciendo así el aprendizaje a través de las vías auditivas (Seppänen et al., 2012; Strait et al., 2013). Así pues, diversos autores han evidenciado que iniciar el entrenamiento musical a temprana edad aumenta el impacto de las adaptaciones cerebrales generadas a nivel estructural y funcional, induciendo más volumen en el córtex auditivo y en la corteza premotora y mejorando la conectividad interhermisférica, velocidad de procesamiento y el rendimiento en procesos motrices, perceptivos y propioceptivos (Alluri et al., 2017; Bailey et al., 2012; Meyer et al., 2018; Reybrouck y Brattico, 2015; Seppänen et al., 2012). Junto con ello, se han observado mejoras en la función auditiva, integración sensomotriz, memoria visual y auditiva y en la inteligencia fluida (Rodrigues et al., 2014; Schlaug et al., 2014; Strait et al., 2013). Todos estos cambios cerebrales, a su vez, repercuten sobre la capacidad de aprendizaje y sobre las habilidades académicas de los niños y niñas en edad escolar (Álvaro-mora y Serrano-rosa, 2019).

A pesar de ello, algunos estudios apuntan que la influencia de diversos factores contextuales como el nivel socioeconómico, nivel de estudios de los padres o la metodología educativa musical, junto con factores personales previos como el coeficiente intelectual o el funcionamiento cognitivo, inciden sobre la posibilidad de iniciar y continuar una formación musical, así como sobre el impacto de la misma (Corrigall et al., 2013; Joret et al., 2017; Linnavalli et al., 2018). En cambio, un estudio longitudinal reciente llevado a cabo con niños de seis años, concluyó que los cambios cerebrales eran atribuidos exclusivamente al entrenamiento musical y no a factores pre-existentes o contextuales (Habibi et al., 2018). A fin de elucidar si los beneficios observados se deben únicamente a la educación musical o si, por el contrario, aspectos biológicos y ambientales juegan un papel decisivo, han ido emergiendo investigaciones prospectivas en la población infantil, adulta y clínica. 


\section{EVIDENCIAS EXPERIMENTALES SOBRE LOS EFECTOS DE LA EDUCA- CIÓN MUSICAL EN LA INFANCIA}

Tal y como se ha comentado previamente, la mayoría de los estudios hasta ahora realizados, al contar con diseños ex post facto, no permiten conocer la causalidad y por ello, resulta esencial continuar investigando mediante diseños experimentales que aporten evidencias sobre las variables involucradas en las modulaciones neurales y neuropsicológicas inducidas por la educación musical (Tervaniemi et al., 2018). Teniendo en cuenta la relevancia que la edad de inicio puede tener y contemplando que las características propias de la infancia pueden favorecer un mayor impacto de la educación musical sobre distintos aspectos neuropsicológicos, cada vez son más los estudios implementados en dicha etapa.

\subsection{Impacto de intervenciones} musicales basadas en el aprendizaje musical instrumental

Partiendo de que la mayoría de las evidencias se han obtenido estudiando el cerebro y rendimiento de aquellas personas que cuentan con una formación musical instrumental y formal, muchos de los estudios experimentales realizados en la infancia se han basado en dicho enfoque. De este modo, y mediante intervenciones de dos años con niños en etapa escolar, se han evidenciado mejoras en el procesamiento auditivo, aumento en la activación neural ante tareas de inhibición cognitiva, desarrollo de la codificación neural del habla, capacidad de discriminación de cambios tonales del habla, procesos del lenguaje y en la fluencia lectora (Habibi et al., 2016, 2018; Kraus et al., 2014). De forma similar, se han hallado mejoras en la memoria de trabajo y en la capacidad viso-perceptiva tras una intervención de tres años con niños de ocho años (Rickard et al., 2010); mejoras en el razonamiento visoespacial, resolución de problemas no verbales, habilidades matemáticas, rendimiento académico y en el bienestar psicosocial tras un año de intervención con niños de entre ocho y 11 años (Osborne et al., 2016); y aumento de la memoria verbal tras 18 meses de entrenamiento musical con niños de entre seis y 12 años (Roden et al., 2012). Junto con ello, investigaciones basadas en intervenciones a corto plazo (máximo seis meses) también han evidenciado efectos positivos en la percepción y procesamiento neural de habla y en la me- moria de trabajo (Guo et al., 2018; Nan et al., 2018).

Por el contrario, también hay estudios que no han hallado efectos positivos a nivel neuronal, cognitivo, emocional, motriz o social (Habibi et al., 2014; Janus et al., 2016; Rickard et al., 2012), lo cual refleja la existencia de evidencias mixtas e inconcluyentes.

\subsection{Impacto de intervenciones musicales basadas en enfoques integralles y multimodales}

Las sociedades occidentales, al centrarse en una noción jerárquica de la música, han ignorado la existencia de diversos modos de relacionarse con la música, donde factores personales y ambientales (cantidad de escucha musical diaria, respuesta emocional o la participación en actividades musicales informales) configuran la experiencia musical de cada persona (Levitin, 2012; Müllensiefen et al., 2014). De este modo, la competencia musical, entendida como la habilidad para percibir, discriminar y recordar melodías y ritmos musicales, estaría mediada también por las complejas interacciones entre factores de la naturaleza y de la crianza (Swaminathan y Schellenberg, 2018).

Así pues, se ha observado que la educación musical no instrumental también promueve modificaciones en las habilidades auditivas y en los procesos neurocognitivos de los niños, a partir de lo cual diversos autores han estudiado el impacto de intervenciones musicales integrales sin implicar el aprendizaje de un instrumento musical (Putkinen et al., 2013, 2015). En esta línea, y respecto a aspectos neurolingüísticos, se han observado mejoras en el desarrollo del lenguaje, en la conciencia fonológica y en el vocabulario de niños de la etapa de Infantil tras dos años de intervención musical basada en juegos y exploración de elementos musicales mediante el movimiento, canto y el ritmo (Herrera et al., 2011 ; LinnavaIli et al., 2018; Lorenzo et al., 2014). Acorde con estos estudios, se han hallado mejoras en la conciencia fonológica de niños de Infantil tras un programa melódico de cuatro meses (Patscheke et al., 2018) y tras una intervención de tan solo 28 dias (Degé y Schwarzer, 2011). Estos últimos autores además destacaron que la música y el lenguaje comparten mecanismos cerebrales para la categorización del sonido. Del mismo modo, también se han halla- 
do mejoras en el razonamiento verbal y en la memoria a corto plazo tras una intervención de 13 semanas (Kaviani et al., 2014) y en la inteligencia verbal tras 20 días de intervención (Moreno et al., 2011), ambos en la primera infancia. Da Silva et al. (2017), por su parte, observaron mejoras en las habilidades numéricas de niños de cinco años mediante la formación musical no instrumental basada en tareas de ritmo, melodía y tono y Barrett et al. (2018) mostraron que los niños de tres y cinco años que realizaban una práctica musical compartida estaban mejor preparados para posteriores experiencias escolares. En este sentido, Benítez et al. (2016) remarcaron que las clases musicales activas promueven un mejor rendimiento que las clases receptivas en tareas de memoria en niños de la etapa de Infantil.

Como puede observarse, la mayoría de los estudios basados en una educación musical más integral y multimodal se han abordado en la etapa de Infantil, lo cual podría entenderse por el enfoque global que caracteriza dicha etapa escolar. Sin embargo, y a pesar de ser pocos, algunos autores también han estudiado el efecto de intervenciones musicales no instrumentales en la etapa de Primaria. Así pues, se han hallado mejoras en diversas habilidades neurocognitivas como la inteligencia verbal, la planificación, la inhibición, la segmentación del habla y la memoria de trabajo mediante intervenciones globales o basadas en pedagogías activas como la de Orff o Kodaly (Chobert et al., 2012; François et al., 2012; Jaschke et al., 2018; Moreno et al., 2009; Roden et al., 2014).

\section{EL VALOR AÑADIDO DE LA CREATIVIDAD MUSICAL}

Tal y como demuestran diversos estudios, la creatividad musical es un complejo proceso cognitivo que se apoya en la musicalidad y en la inteligencia (Oikkonen et al., 2016). Según Bashwiner et al. (2016), el bagaje musical, la experiencia emocional ante la música y el estilo de procesamiento cognitivo inciden sobre el proceso creativo musical. La composición y la improvisación son dos importantes actividades musicales que requieren de una interconexión bidireccional entre el pensamiento convergente y divergente, y de la integración de procesos emocionales, cognitivos y motivacionales ( $\mathrm{Vi}$ llarreal et al., 2013; Wong y Lim, 2017). Junto con ello, ambas son actividades creativas que favorecen el desarrollo de la memoria emocional, las relaciones interpersonales y una mayor conectividad funcional entre la corteza prefrontal dorsolateral bilateral, el área premotora dorsal y el área suplementaria (Diaz-Abrahan et al., 2018; Passanisi et al., 2015; Pinho et al., 2014).

La capacidad creativa está presente en todo ser humano, pero factores genéticos y biológicos favorecen una predisposición natural para la capacidad de resolución de problemas en distintos ámbitos (Şahin, 2016). De esto modo, la capacidad creativa varía de una persona a otra del mismo modo que también lo hace a lo largo del desarrollo evolutivo, presentando una tendencia ascendente entre los ocho y 11 años y descendente a partir de los 12 años (Sastre-Riba y Pascual-Sufrate, 2013; Stevenson et al., 2014). Por ello, resulta clave incidir en el desarrollo de la capacidad creativa durante la etapa escolar. Diversas investigaciones han demostrado que las tareas creativo-musicales promueven el desarrollo del pensamiento creativo, la imaginación, la flexibilidad, la originalidad y de las relaciones sociales en niños de la etapa escolar (Corakli y Batibay, 2012; Koutsoupidou y Hargreaves, 2009; Passanisi et al., 2015). Por ello, y partiendo de que el desarrollo creativo es mayor en la etapa de Primaria, la actividad creativo-musical puede ser un motivante y enriquecedor recurso que potencie la creatividad, favoreciendo, a su vez, mejoras en el rendimiento escolar (Gajda et al., 2017; Lamana-Selva y De-La-Peña, 2018).

\section{CONCLUSIONES}

La presencia universal y diaria de la música en la vida de las personas, su implicación en el desarrollo humano y ontogenético, y su poder para modular y activar simultáneamente procesos perceptivos, emocionales y cognitivos, ponen de manifiesto la relevancia que el estudio de la música tiene para la investigación neurocientífica y neuropsicológica, y, del mismo modo, también para la comunidad educativa y para la sociedad en general (Benítez et al., 2018; Pearce y Rohrmeier, 2012; Tervaniemi et al., 2018).

Como se ha podido observar, la literatura muestra resultados dispares, así como pocas evidencias respecto al impacto de intervenciones musicales no instrumentales en la etapa de Primaria y menos aún, las asociadas al 
efecto de la creatividad musical. Esto pone de manifiesto la necesidad de continuar investigando en este ámbito mediante estudios experimentales, con el objetivo principal de cubrir la escasez de evidencias respecto al impacto de una educación musical global, multimodal e integrada de procesos creativo-musicales, sobre los procesos neuropsicológicos y el rendimiento académico de niños de la etapa de Primaria.

Para futuros estudios sería conveniente contemplar las limitaciones detectadas por otros autores como garantizar la representatividad y aleatorización de la muestra, contar con un grupo de control pasivo y otro de control activo, prever un porcentaje de posibles abandonos, detallar los programas implementados y controlar variables ambientales y personales previas que pudieran incidir en los resultados (Álvaro-mora y Serrano-rosa, 2019; Benz et al., 2016; Corrigall y Schellenberg, 2015; Huotilainen y Tervaniemi, 2018). Otro de los aspectos que está aún sin resolver, debido a la contradicción existente en los resultados de distintos estudios, es la duración de las intervenciones. Algunos autores señalan que para obtener impactos positivos es necesario realizar intervenciones de más de seis semanas de duración (Mehr et al., 2013), otros defienden que estas no tienen que ser muy extensas aunque sí intensivas y con una duración mínima de seis meses (Chobert et al., 2012) otros consideran que un año es insuficiente (Kraus et al. 2014) y en este sentido, Linnavalli et al. (2018) establecen un mínimo de dos años para observar diferencias en las respuestas cerebrales. Controlar todos estos factores, permitirá elucidar las diversas contradicciones existentes en relación a la edad más adecuada, y a la duración y tipo de intervención necesaria para obtener resultados positivos en aspectos neuroanatómicos y funcionales, así como en las transferencias cognitivas hacia los procesos académicos.

Así pues, la mayoría de estudios apuntan hacia un impacto positivo de la educación musical sobre distintos procesos neuropsicológicos y académicos, pero es necesario continuar aportando evidencias respecto a niños en edad escolar (6-12 años). Conocer las relaciones causales asociadas a cada habilidad académica, permitiría diseñar e implementar programas musicales transversales en el ámbito escolar, orientándolos a la mejora de cada habilidad específica y fomentando, a su vez, mejoras en el rendimiento académico de cada alumno.

\section{REFERENCIAS}

Alluri, V., Toiviainen, P., Burunat, I., Kliuchko, M., Vuust, P. y Brattico, E. (2017). Connectivity patterns during music listening: Evidence for action-based processing in musicians. Human Brain Mapping, 38(6), 2955-2970. https://doi.org/10.1002/hbm.23565

Álvaro-mora, C. y Serrano-rosa, M. Á. (2019). Influencia de la formación musical en el rendimiento académico: una revisión bibliográfica. Anvario de Psicología, 49(1), 18-31. https://doi.org/10.1344/anpsic2019.49.3

Bailey, J. A., Zatorre, R. J. y Pehune, Virginia, B. (2012). Early Musical Training Is Linked to Gray Matter Structure in the Ventral Premotor Cortex and Auditory-Motor Rhythm Synchronization Performance. Journal of Cognitive Neuroscience, 24(6), 1275-1285. https://doi.org/10.1162/jocn

Barrett, M. S., Flynn, L. M. y Welch, G. F. (2018). Music value and participation: An Australian case study of music provision and support in Early Childhood Education. Research Studies in Music Education, 40(2), 226-243. https:// doi.org/10.1177/1321103X18773098

Bashwiner, D. M., Wertz, C. J., Flores, R. A. y Jung, R. E. (2016). Musical Creativity Revealeda in Brain Structure: Interplay between Motor, Default Mode, and Limbic Networks. Scientific Reports, 6(20482), 1-8. https:// doi.org/10.1038/srep20482

Benítez, Maria A., Díaz-Abrahan, V. y Nadia, J. (2016). Efecto del entrenamiento musical en la memoria emocional de niños en edad prescolar. Efecto Del Entrenamiento Musical En La Memoria Emocional de Niños En Edad Prescolar. Xii Congreso Argentino de Neuropsicología.

Benítez, María Angélica, Díaz-Abrahan, V. y Justel, N. (2018). Beneficios del entrenamiento musical en el desarrollo infantil: una revisión sistemática. Revista Internacional de Educación Musical, 5, 61-69. https://doi. org/10.12967/riem-2017-5-p061-069

Benz, S., Sellaro, R., Hommel, B. y Colzato, L. S. (2016). Music makes the world go round: The impact of musical training on non-musical cognitive functions-a review. Frontiers in Psychology, 6, 1-5. https://doi. org/10.3389/fpsyg.2015.02023 
Bidelman, G. M. y Krishnan, A. (2010). Effects of reverberation on brainstem representation of speech in musicians and non-musicians. Brain Research, 1355, 112-125. https://doi.org/doi:10.1016/j.brainres.2010.07.100.

Boone, W. y Piccinini, G. (2016). The cognitive neuroscience revolution. Synthese, 193(5), 1509-1534. https://doi.org/10.1007/ s $11229-015-0783-4$

Burunat, I., Brattico, E., Puoliväli, T., Ristaniemi, T., Sams, M. y Toiviainen, P. (2015). Action in Perception: Prominent Visuo-Motor Functional Symmetry in Musicians during Music Listening. Plos One, 10(9), e0138238. https://doi.org/10.1371/journal.pone.0138238

Burunat, I., Tsatsishvili, V., Brattico, E. y Toiviainen, P. (2017). Coupling of Action-Perception Brain Networks during Musical Pulse Processing: Evidence from Region-of-Interest-Based Independent Component Analysis. Frontiers in Human Neuroscience, 77 (230), 1-14. https://doi.org/10.3389/ fnhum.2017.00230

Carmona, C., Sánchez, P. y Bakieva, M. (2011). Actividades Extraescolares y Rendimiento Académico: Diferencias en Autoconcepto y Género. Revista de Investigación Educativa, 29(2), 447-465.

Chobert, J., François, C., Velay, J. L. y Besson, M. (2012). Twelve months of active musical training in 8-to 10-year-old children enhances the preattentive processing of syllabic duration and voice onset time. Cerebral Cortex, 24(4), 956-967. https://doi. org/10.1093/cercor/bhs377

Corakli, E. y Batibay, D. (2012). The Efficacy of a Music Education Programme Focused on Creative Thinking. Procedia - Social and Behavioral Sciences, 46, 3571-3576. https:// doi.org/10.1016/i.sbspro.2012.06.107

Corrigall, K. A. y Schellenberg, E. G. (2015). Predicting who takes music lessons: Parent and child characteristics. Frontiers in Psychology, 6(282), 1-8. https://doi.org/10.3389/ fpsyg.2015.00282

Corrigall, K. A., Schellenberg, E. G. y Misura, N. M. (2013). Music Training, Cognition, and Personality. Frontiers in Psychology. https://doi.org/10.3389/fpsyg.2013.00222

Da Silva, E. R., Dos Santos Baldin, M. y Dos Santos, F. H. (2017). Cognitive effects of numeracy musical training in brazilian preschool children: A prospective pilot study. Psychology and Neuroscience, 10(3), 281-296. https://doi.org/10.1037/pne0000098

Degé, F. y Schwarzer, G. (2011). The effect of a music program on phonological awareness in preschoolers. Frontiers in Psychology, 2, 124. https://doi.org/10.3389/ fpsyg.2011.00124

Diaz-Abrahan, V. M., Shifres, F. y Justel, N. R. (2018). Music improvisation modulates emotional memory. Psychology of Music, 1-16. https://doi. org/10.1177/0305735618810793

Fauvel, B., Groussard, M., Chételat, G., Fouquet, M., Landeau, B., Eustache, F., Desgranges, B. y Platel, H. (2014). Morphological brain plasticity induced by musical expertise is accompanied by modulation of functional connectivity at rest. Neurolmage, 90, 179-188. https://doi.org/10.1016/j. neuroimage.2013.12.065

Filippetti, V. A. (2011). Funciones ejecutivas en niños escolarizados: efectos de la edad y el estrato socioeconomico. Avances En Psicologia Latinoamericana, 29(1), 98-113.

François, C., Chobert, J., Besson, M. y Schön, D. (2012). Music training for the development of speech segmentation. Cerebral Cortex, 23(9), 2038-2043. https://doi. org/10.1093/cercor/bhs 180

Gajda, A., Karwowski, M. y Beghetto, R. A. (2017). Creativity and academic achievement: A meta-analysis. Journal of Educational Psychology, 109(2), 269-299.

Galera Núñez, M. del M. y Tejada Giménez, J. (2012). Lectura musical y procesos cognitivos implicados. LEEME: Lista Electrónica Europea de Música En La Educación, 29, 59-82. http://musica.rediris.es/leeme

Guo, X., Ohsawa, C., Suzuki, A. y Sekiyama, K. (2018). Improved Digit Span in children after a 6 -week intervention of playing a musical instrument: An exploratory randomized controlled trial. Frontiers in Psychology, 
8(2303), 1-9. https://doi.org/10.3389/ fpsyg.2017.02303

Habibi, A., Cahn, B. R., Damasio, A. y Damasio, H. (2016). Neural correlates of accelerated auditory processing in children engaged in music training. Developmental Cognitive Neuroscience, 21 (2016), 1-14. https://doi. org/10.1016/j.den.2016.04.003

Habibi, A., Damasio, A., Ilari, B., Sachs, M. E. y Damasio, H. (2018). Music training and child development: A review of recent findings from a longitudinal study. Annals of the New York Academy of Sciences, 1423(1), 73-81. https://doi.org/10.1111/ nyas. 13606

Habibi, A., Ilari, B., Crimi, K., Metke, M., Kaplan, J. T., Joshi, A. A., Leahy, R. M., Shattuck, D. W., Choi, S. Y., Haldar, J. P., Ficek, B., Damasio, A. y Damasio, H. (2014). An equal start: absence of group differences in cognitive, social, and neural measures prior to music or sports training in children. Frontiers in Human Neuroscience, 8(690), 1-11. https://doi.org/10.3389/fnhum.2014.00690

Hallam, S. (2010). The power of music: Its impact on the intellectual, social and personal development of children and young people. International Journal of Music Education, 28(3), 269-289. https://doi. org/10.1177/0255761410370658

Herholz, S. C. y Zatorre, R. J. (2012). Musical Training as a Framework for Brain Plasticity: Behavior, Function, and Structure. Neuron, 76(3), 486-502. https://doi.org/https:// doi.org/10.1016/i.neuron.2012.10.011

Herrera, L., Lorenzo, O., Defior, S., Fernandez-Smith, G. y Costa-Giomi, E. (2011). Effects of phonological and musical training on the reading readiness of native- and foreign-Spanish-speaking children. Psychology of Music, 39(1), 68-81. https://doi. org/10.1177/0305735610361995

Huotilainen, M. y Tervaniemi, M. (2018). Planning music-based amelioration and training in infancy and childhood based on neural evidence. Annals of the New York Academy of Sciences, 1423, 146-154. https://doi. org/10.1111/nyas. 13655
James, C. E., Oechslin, M. S., Michel, C. M. y Pretto, M. De. (2017). Electrical neuroimaging of music processing reveals mid-latency changes with level of musical expertise. Frontiers in Neuroscience, $17(613)$, 1-19. https://doi.org/10.3389/ fnins.2017.00613

James, C. E., Oechslin, M. S., Van De Ville, D., Havert, C.-A., Descloux, C. y Lazeyras, F. (2013). Musical training intensity yields opposite effects on grey matter density in cognitive versus sensorimotor networks. Brain Structure and Function, 219(1), 353-366. https://doi.org/10.1007/s00429-0130504-z

Janus, M., Lee, Y., Moreno, S. y Bialystok, E. (2016). Effects of short-term music and second-language training on executive control. Journal of Experimental Child Psychology, 144, 84-97. https://doi.org/10.1016/j. jecp.2015.11.009

Jaschke, A. C., Honing, H. y Scherder, E. J. A. (2018). Longitudinal Analysis of Music Education on Executive Functions in Primary School Children. Frontiers in Neuroscience, 12(103), 1-11. https://doi.org/10.3389/ fnins.2018.00103

Jentschke, S., Friederici, A. D. y Koelsch, S. (2014). Neural correlates of music-syntactic processing in two-year old children. Developmental Cognitive Neuroscience, 9, 200-208. https://doi.org/10.1016/i. den.2014.04.005

Joret, M. E., Germeys, F. y Gidron, Y. (2016). Cognitive inhibitory control in children following early childhood music education. Musicae Scientiae, 21 (3), 303-315. https:// doi.org/10.1177/1029864916655477

Kaviani, H., Mirbaha, H., Pournaseh, M. y Sagan, O. (2014). Can music lessons increase the performance of preschool children in IQ tests? Cognitive Processing, 15(1), 77-84. https://doi.org/https://doi.org/10.1007/ s10339-013-0574-0

Koelsch, S. (2009). Neural substrates of processing syntax and semantics in music. Music That Works: Contributions of Biology, Neurophysiology, Psychology, Sociology, Medicine and Musicology, 143-153. https://doi. org/https://doi.org/10.1007/978-3-211 $75121-39$ 
Koutsoupidou, T. y Hargreaves, D. J. (2009). An experimental study of the effects of improvisation on the development of children's creative thinking in music. Psychology of Music, 37(3), 251-278. https://doi. org/10.1177/0305735608097246

Kraus, N., Hornickel, J., Strait, D. L., Slater, J. y Thompson, E. (2014). Engagement in community music classes sparks neuroplasticity and language development in children from disadvantaged backgrounds. Frontiers in Psychology, 5(1403), 1-9. https://doi. org/10.3389/fpsyg.2014.01403

Kraus, N., Slater, J., Thompson, E. C., Hornickel, J., Strait, D. L., Nicol, T. y White-Schwoch, T. (2014). Music Enrichment Programs Improve the Neural Encoding of Speech in At-Risk Children. Journal of Neuroscience, 34(36), 11913-11918. https://doi.org/ doi:10.1523/jneurosci.1881-14.2014

Lamana-Selva, M. . y De-La-Peña, C. (2018). Rendimiento Académico En Matemáticas: relación con creatividad y estilos de afrontamiento. Revista Mexicana de Investigación Educativa RMIE, 23(79), 1075-1092.

Levitin, D. J. (2012). What Does It Mean to Be Musical? Neuron, 73(4), 633-637. https:// doi.org/10.1016/i.neuron.2012.01.017

Linnavalli, T., Putkinen, V., Lipsanen, J., Huotilainen, M. y Tervaniemi, M. (2018). Music playschool enhances children's linguistic skills. Scientific Reports, 8(1), 1-10. https:// doi.org/10.1038/s41598-018-27126-5

Lorenzo, O., Herrera, L., Hernández-Candelas, M. y Badea, M. (2014). Influence of Music Training on Language Development. A Longitudinal Study. Procedia - Social and Behavioral Sciences, 128, 527-530. https://doi. org/10.1016/j.sbspro.2014.03.200

Marco-Pallarés, J., Martínez-Molina, N., Mas-Herrero, E., Rodríguez-Fornells, A. y Zatorre, R. J. (2016). Neural correlates of specific musical anhedonia. Proceedings of the National Academy of Sciences, $173(46)$, E7337-E7345. https://doi.org/10.1073/ pnas. 1611211113

Maureira, F. (2010). La neurociencia cognitiva ¿una ciencia base para la psicología? Psiquiatría Universitaria, 6(4), 449-453.
Mehr, S. A., Schachner, A., Katz, R. C. y Spelke, E. S. (2013). Two randomized trials provide no consistent evidence for nonmusical cognitive benefits of brief preschool music enrichment. PLoS ONE, 8(12), e82007. https:// doi.org/10.1371/journal.pone.0082007

Meyer, J., Oguz, P. G. y Moore, K. S. (2018). Superior fluid cognition in trained musicians. Psychology of Music, 48(3), 1-14. https:// doi.org/10.1177/0305735618808089

Moreno, S., Bialystok, E., Barac, R., Schellenberg, E. G., Cepeda, N. J. y Chau, T. (2011). Short-term music training enhances verbal intelligence and executive function. Psychological Science, 22(11), 1425-1433. https:// doi.org/10.1177/0956797611416999

Moreno, S. y Bidelman, G. M. (2014). Examining neural plasticity and cognitive benefit through the unique lens of musical training. Hearing Research, 308, 84-97. https://doi. org/10.1016/j.heares.2013.09.012

Moreno, S., Marques, C., Santos, A., Santos, M., Castro, S. L. y Besson, M. (2009). Musical training influences linguistic abilities in 8 -year-old children: More evidence for brain plasticity. Cerebral Cortex, 19(3), 712-723. https://doi.org/10.1093/cercor/bhn 120

Müllensiefen, D., Gingras, B., Musil, J. y Stewart, L. (2014). The musicality of non-musicians: An index for assessing musical sophistication in the general population. PLOS ONE, 9(2), e89642. https://doi.org/10.1371/journal. pone.0089642

Nan, Y., Liu, L., Geiser, E., Shu, H., Gong, C. C., Dong, Q., Gabrieli, J. D. E. y Desimone, R. (2018). Piano training enhances the neural processing of pitch and improves speech perception in Mandarin-speaking children. Proceedings of the National Academy of Sciences, 115(28), E6630-E6639. https://doi. org/10.1073/pnas. 1808412115

Oikkonen, J., Kuusi, T., Peltonen, P., Raijas, P., Ukkola-Vuoti, L., Karma, K., Onkamo, P. y Järvelä, I. (2016). Creative activities in music - A genome-wide linkage analysis. PLoS ONE, $17(2)$, e0148679. https://doi. org/10.1371/journal.pone.0148679

Osborne, M. S., McPherson, G. E., Faulkner, R., Davidson, J. W. y Barrett, M. S. (2016). 
Exploring the academic and psychosocial impact of El Sistema-inspired music programs within two low socio-economic schools. Music Education Research, 18(2), 156-175. https://doi.org/10.1080/14613 808.2015 .1056130

Passanisi, A., Di Nuovo, S., Urgese, L. y Pirrone, C. (2015). The Influence of Musical Expression on Creativity and Interpersonal Relationships in Children. Procedia - Social and Behavioral Sciences, 191, 24762480 . https://doi.org/10.1016/i.sbspro.2015.04.308

Patscheke, H., Degé, F. y Schwarzer, G. (2018). The effects of training in rhythm and pitch on phonological awareness in four- to six-year-old children. Psychology of Music, 47(3), 1-16. https://doi. org/10.1177/0305735618756763

Pearce, M. y Rohrmeier, M. (2012). Music Cognition and the Cognitive Sciences. Topics in Cognitive Science, 4(4), 468484. https://doi.org/10.1111/i.17568765.2012.01226.x

Penhune, V. B. (2011). Sensitive periods in human development: Evidence from musical training. Cortex, 47(9), 1126-1137. https:// doi.org/10.1016/i.cortex.2011.05.010

Pinho, A. L., de Manzano, O., Fransson, P., Eriksson, H. y Ullen, F. (2014). Connecting to Create: Expertise in Musical Improvisation Is Associated with Increased Functional Connectivity between Premotor and Prefrontal Areas. Journal of Neuroscience, 34(18), 6156-6163. https://doi.org/10.1523/ jneurosci.4769-13.2014

Portellano, J. A. (2008). Neuropsicología infantil. Editoria Síntesis S.A.

Portellano, J. A. (2010). Introducción a la neuropsicología. McGraw Hil.

Putkinen, V., Saarikivi, K. y Tervaniemi, M. (2013). Do informal musical activities shape auditory skill development in preschool-age children? Frontiers in Psychology, 4(572), 1-6. https://doi.org/10.3389/ fpsyg.2013.00572

Putkinen, V., Tervaniemi, M., Saarikivi, K. y Huotilainen, M. (2015). Promises of formal and informal musical activities in advancing neurocognitive development throughout childhood. Annals of the New York Academy of Sciences, 1337(1), 153-162. https://doi. org/10.1111/nyas. 12656

Reybrouck, M. (2010). Music cognition and real-time listening: denotation, cue abstraction, route description and cognitive maps. Musicæ Scientiæ, 14(2), 187-202.

Reybrouck, M. y Brattico, E. (2015). Neuroplasticity beyond Sounds: Neural Adaptations Following Long-Term Musical Aesthetic Experiences. Brain Sciences, 5(1), 69-91. https://doi.org/10.3390/brainsci5010069

Rickard, N. S., Bambrick, C. J. y Gill, A. (2012). Absence of widespread psychosocial and cognitive effects of schoolbased music instruction in 10-13-year-old students. International Journal of Music Education, 30(1), 57-78. https://doi. org/10.1177/0255761411431399

Rickard, N. S., Vasquez, J. T., Murphy, F., Gill, A. y Toukhsati, S. R. (2010). Benefits of a classroom based instrumental music program on verbal memory of primary school children: a longitudinal study. Australian Journal of Music Education, 1, 36-47.

Roden, I., Grube, D., Bongard, S. y Kreutz, G. (2014). Does music training enhance working memory performance? Findings from a quasi-experimental longitudinal study. Psychology of Music, 42(2), 284-298. https:// doi.org/10.1177/0305735612471239

Roden, I., Kreutz, G. y Bongard, S. (2012). Effects of a school-based instrumental music program on verbal and visual memory in primary school children: A longitudinal study. Frontiers in Psychology, 3(572), 1-9. https:// doi.org/10.3389/fpsyg.2012.00572

Rodrigues, A. C., Loureiro, M. y Caramelli, P. (2014). Visual memory in musicians and non-musicians. Frontiers in Human Neuroscience, 8(424), 1-10. https://doi. org/10.3389/fnhum.2014.00424

Saari, P., Burunat, I., Brattico, E. y Toiviainen, P. (2018). Decoding Musical Training from Dynamic Processing of Musical Features in the Brain. Scientific Reports, 8(1), 1-12. https:// doi.org/10.1038/s41598-018-19177-5 
Şahin, F. (2016). General intelligence, emotional intelligence and academic knowledge as predictors of creativity domains: A study of gifted students. Cogent Education, 3(1), 1-17. https://doi.org/10.1 080/2331 186X.2016.1218315

Sanju, H. K. y Kumar, P. (2016). Enhanced auditory evoked potentials in musicians: $A$ review of recent findings. Journal of Otology, $17(2)$, 63-72. https://doi.org/10.1016/i. joto.2016.04.002

Sastre-Riba, S. y Pascual-Sufrate, M. T. (2013). Alta capacidad intelectual, resolución de problemas y creatividad. Revista de Neurologia, 56(1), 67-76.

Schlaug, G., Forgeard, M., Zhu, L., Norton, A., Norton, A. y Winner, E. (2014). Training-induced Neuroplasticity in Young Children. Annals of the New York Academy of Sciences, 1 169, 205-208. https://doi.org/ doi:10.1111/i.1749-6632.2009.04842.x.

Seppänen, M., Hämäläinen, J., Pesonen, A.K. y Tervaniemi, M. (2012). Music Training Enhances Rapid Neural Plasticity of N1 and P2 Source Activation for Unattended Sounds. Frontiers in Human Neuroscience, 6(43), 1-13. https://doi.org/10.3389/fnhum.2012.00043

Skoe, E. y Kraus, N. (2013). Musical training heightens auditory brainstem function during sensitive periods in development. Frontiers in Psychology, 4(622), 1-15. https://doi. org/10.3389/fpsyg.2013.00622

Slater, J., Azem, A., Nicol, T., Swedenborg, B. y Kraus, N. (2017). Variations on the theme of musical expertise: cognitive and sensory processing in percussionists, vocalists and non-musicians. European Journal of Neuroscience, 45, 952-963. https://doi. org/10.1111/ejn.13535

Stevenson, C. E., Kleibeuker, S. W., de Dreu, C. K. W. y Crone, E. A. (2014). Training creative cognition: adolescence as a flexible period for improving creativity. Frontiers in Human Neuroscience, 8(827), 1-16. https://doi. org/10.3389/fnhum.2014.00827

Strait, D. L., Parbery-Clark, A., O'Connell, S. y Kraus, N. (2013). Biological impact of preschool music classes on processing speech in noise. Developmental Cognitive Neuroscience, 6, 51-60. https://doi.org/10.1016/i. dcn.2013.06.003

Swaminathan, S. (2018). Nonmusical correlates of musical ability. University of Toronto.

Swaminathan, S. y Schellenberg, E. G. (2018). Musical Competence is Predicted by Music Training, Cognitive Abilities, and Personality. Scientific Reports, 6(43), 1-7. https://doi. org/10.1038/s41598-018-27571-2

Tanaka, S. y Kirino, E. (2018). The parietal opercular auditory-sensorimotor network in musicians: A resting-state fMRI study. Brain and Cognition, 120, 43-47. https://doi. org/10.1016/j.bandc.2017.11.001

Tervaniemi, M., Tao, S. y Huotilainen, M. (2018). Promises of Music in Education? Frontiers in Education, 3(74), 1-6. https:// doi.org/10.3389/feduc.2018.00074

Villarreal, M. F., Cerquetti, D., Caruso, S., Schwarcz López Aranguren, V., Gerschcovich, E. R., Frega, A. L. y Leiguarda, R. C. (2013). Neural Correlates of Musical Creativity: Differences between High and Low Creative Subjects. PLoS ONE, 8(9), e75427. https://doi.org/10.1371/journal. pone.0075427

Vollmann, $H_{\text {., Ragert, }}$., Conde, V., Villringer, A., Classen, J., Witte, O. W. y Steele, C. J. (2014). Instrument specific use-dependent plasticity shapes the anatomical properties of the corpus callosum: a comparison between musicians and non-musicians. Frontiers in Behavioral Neuroscience, 8(245), 1-8. https://doi.org/10.3389/fnbeh.2014.00245

Winner, E., Goldstein, T. G. y Vincent-Lancrin, S. (2013). Arts for arts's sake? The impact of arts education. OECD Publishing.

Wong, S. S. H. y Lim, S. W. H. (2017). Mental imagery boosts music compositional creativity. PLoS ONE, 12(3), e0174009. https:// doi.org/10.1371/journal.pone.0174009 